\title{
Current Understanding on Perioperative Management in Lung Cancer: Implications for Anesthetic Considerations
}

This article was published in the following Dove Press journal:

Drug Design, Development and Therapy

\author{
Jian Wang \\ Lei Liu (1D ${ }^{2}$ \\ Yuli Song $\mathbb{1}^{3}$ \\ Jinghua Jiao $\mathbb{D}^{4}$ \\ Yifan Zhong ${ }^{2}$
}

'Department of Anesthesiology, First Hospital of China Medical University, Shenyang, Liaoning, I I000I, People's Republic of China; ${ }^{2}$ Department of Ophthalmology, First Hospital of China Medical University, Shenyang, Liaoning, I I000I, People's Republic of China;

${ }^{3}$ Department of Gynaecology, The First Affiliated Hospital of China Medical University, Shenyang, Liaoning, I I000I, People's Republic of China; ${ }^{4}$ Department of Anesthesiology, Central Hospital, Shenyang Medical College, Shenyang, I I 0024, People's Republic of China
Correspondence: Jinghua Jiao

Department of Anesthesiology, Central Hospital, Shenyang Medical College,

Shenyang, I I0024, People's Republic of

China

Email jean0905@I63.com

Yifan Zhong

Department of Ophthalmology, First Hospital of China Medical University,

Shenyang, Liaoning, I I000I, People's

Republic of China

Email zhongyifan85IIII@I63.com

\begin{abstract}
Narcotic drugs are often used to treat perioperative pain for patients with lung cancer. However, anesthetic management and narcotic substance use may have significant impacts on patients with lung cancer, including anti-cancer or promoting cancer effects. In this study, we summarize the effects of anesthetic management and its related substances on lung cancer. An evidence-based review of the influence of anesthetic techniques and narcotic substances used on lung cancer was performed. The effects of perioperative pain management and the method of choosing anesthesia for patients with lung cancer were explored. Different management techniques of anesthesia have been indicated to suppress both cellmediated immunity and humoral immunity and have effects on the recurrence and metastasis of lung cancer. Evidence suggests that the effects of narcotic substances used on lung cancer were still inconsistent. However, the mechanisms by which anesthetics and analgesics inhibit the tumor are complicated. Perioperative management leads to decreased immunity in patients with lung cancer, which to some extent contributes to recurrence and metastasis. Various narcotic substances used may modulate signal pathways, including the mitochondrial pathway, and appear to exert different effects on the recurrence and metastasis of lung cancer. The anesthesiologists should consider these effects on perioperative management with lung cancer.
\end{abstract}

Keywords: narcotic substance use, anesthetics, lung cancer

\section{Introduction}

Lung cancer has become one of the most commonly occurring types of cancer in the world. ${ }^{1}$ Most patients with lung cancer are treated with surgical removal of the primary lesion and lymph node dissection, but some patients still have recurrence and metastasis of lung cancer after surgery. Perioperative traumatic stress and other factors lead to decreased immunity in patients with lung cancer, which to some extent contributes to the recurrence and metastasis. Furthermore, different narcotic substances used have different effects on the recurrence and metastasis of lung cancer. The aim of this review is to summarize the influence of anesthetic techniques and narcotic substances used on lung cancer and provide possible implications in the prevention of cancer.

\section{Searching and Review}

A systematic review of English and Chinese literature was conducted and articles regarding lung cancer, anesthetic management and narcotic substances used were 
obtained. This review was completed by searching in PubMed, Web of science, China National Knowledge Infrastructure (CNKI), Wanfang, and Chongqing VIP databases using the keywords mentioned above. The careful review of the literature was according to the preference and clinical expertise of the authors.

\section{Types of Anesthesia}

Surgery is the most common first-line treatment for most cancers including lung cancer. Anesthesia is most important for lung cancer patients undergoing surgical removal. Xu et $\mathrm{al}^{2}$ compared the effects of total intravenous anesthesia (TIVA) and general anesthesia combined with epidural anesthesia on the cellular immunity and prognosis of patients undergoing thoracoscopic radical resection of non-small cell lung cancer (NSCLC). After the surgery, with either of the anesthesia methods, the number of $\mathrm{T}$ lymphocytes such as $\mathrm{CD} 3(+)$, CD4(+) and CD8(+) T cell and the natural killer cells CD56 $(+)$ in the patients all decreased compared with the preoperative state, but the number of T lymphocytes and natural killer cells in the patients with general anesthesia combined with epidural anesthesia was higher than that in patients with TIVA. It was suggested that general anesthesia combined with epidural anesthesia had less impact on the cellular immune function of patients than TIVA. During the 3-year follow-up, there were recurrences and metastasis of lung cancer after surgery in patients with either TIVA or general anesthesia combined with epidural anesthesia, and there was no significant difference in the recurrence rate and metastasis rate between the two groups of patients. $\mathrm{Fu}$ et $\mathrm{al}^{3}$ pointed out that $\mathrm{CD} 3(+), \mathrm{CD} 4(+)$ and $\mathrm{CD} 25(+) \mathrm{T}$ cells all decreased in patients undergoing radical resection of the lung(s) under TIVA or general anesthesia combined with epidural anesthesia, but the number of these cells in the patients with general anesthesia combined with epidural anesthesia was more than that in the patients with TIVA, suggesting that general anesthesia combined with epidural anesthesia could maintain more stable immune functions after surgery than TIVA. Cata et $\mathrm{al}^{4}$ found that the immune functions of patients with NSCLC was suppressed after thoracotomy, also, the immune functions could not be improved by the use of postoperative epidural analgesia. None of the above studies pointed out that the effect of general anesthesia combined with epidural anesthesia on the recurrence and metastasis of lung cancer after radical resection was better than that of TIVA. In a retrospective cohort study of 1729 patients with NSCLC undergoing thoracotomy, the researchers found that postoperative intravenous analgesia, thoracic epidural analgesia and thoracic paravertebral nerve block for analgesia were not associated with lung cancer recurrence. ${ }^{5}$ However, the long-term survival rate of patients with thoracic paravertebral nerve block for analgesia was higher than that of patients with the other two postoperative analgesic methods, which suggested that general anesthesia combined with regional nerve block might have a positive effect on the overall survival rate of lung cancer patients, but prospective randomized controlled trials are still required to verify these three analgesic methods.

In addition, previous evidence suggested that local anesthesia may have anti-immune properties, which may have impact on lung cancer. ${ }^{6}$ Local anesthesia acts on $\mathrm{G}$ protein-coupled receptors (GPCRs), also called seven transmembrane domain (7TM) proteins, representing the largest family of cell surface receptors. ${ }^{7,8}$ Many critical mediators of immunity act through GPCRs [interleukins (ILs), and lysophosphatidic acid, etc. $],{ }^{9}$ thus, there exists a potential interaction between local anesthesia and the immune response. Local anesthesia attenuates excessive responses of the inflammatory system even in patients with NSCLC.

\section{Narcotic Substances}

\section{Midazolam}

The perioperative management has been established as a potential pathway in influencing tumor microenvironments, with narcotic substances used for anesthesia implicated in affecting the behavior of tumor cells. ${ }^{10-12}$ Midazolam, as a narcotic agent, is commonly used to provide sedation prior to procedures such as laceration repair and reduction of dislocations. Wang et $\mathrm{al}^{13}$ found in human NSCLC cultured in vitro that Midazolam could inhibit the migration of lung cancer cells and induce lung cancer cell apoptosis through the mitochondrial pathway. After implanting the cells of human NSCLC into nude mice, Midazolam significantly reduced the growth of lung cancer cells in nude mice compared with the normal saline control group. The effect of Midazolam on the recurrence and metastasis of lung cancer cells requires further in vivo studies. Furthermore, our previous study also revealed that Midazolam might be a putative anti-cancer approach for NSCLC therapy through inducing apoptosis of A549 cells by targeting signal transduction and activation of transcription 3 (STAT3) via miR-520d-5p. ${ }^{14}$ In vivo, we established the nude mice model of human lung cancer by A549, and found that Midazolam can significantly inhibit the growth of A549 transplanted tumor tissue and induce tumor cell apoptosis by regulating STAT3 expression. ${ }^{15}$ Although there is no clinical-based evidence on Midazolam use for anti-tumor growth, it provides novel insight for future therapeutic strategies against lung cancer. 


\section{Opioids}

For NSCLC patients undergoing surgery or cancer pain management, opioids such as morphine, fentanyl, and oxycodone are the most widely used and effective analgesics. ${ }^{16}$ Previously, many classical opioid receptors, including $\mathrm{mu}(\mu)$, delta $(\delta)$, and kappa $(\kappa)$, have been reported to be expressed in NSCLC cell lines and tumor tissues, and play an important role in cancer outcomes. ${ }^{17}$ Both In vivo and in vitro studies have found that overexpression of opioid receptors increases the growth and metastasis of H358 cell lines (human bronchioloalveolar carcinoma). Based on lung cell specimens of patients with NSCLC, Singleton et al pointed out that the expression level of opioid receptors in patients with lymph node metastasis of lung cancer cells was nearly twice that of patients without metastasis. ${ }^{18}$ Furthermore, the opioid growth factor receptor (OGFR), a receptor of endogenous opioid peptide [Met5]-enkephalin, which is a non-classical opioid receptor, was expressed in both in vivo and vitro models involved in suppression of lung cancer proliferation effects of morphine. ${ }^{19}$ In a retrospective study of 901 patients, Cata et al found that $10.15 \mathrm{ug} / \mathrm{kg}$ equivalent dose of fentanyl on average used in the surgery was a risk factor for the overall survival rate of patients with stage I nonsmall cell lung cancer within 5 years. ${ }^{20}$ The intraoperative administration of opioids was related to the decline of overall survival rate of patients with stage I non-small cell lung cancer after surgery, but it had nothing to do with the overall survival rate of patients with stage II and III NSCLC. In a single-center retrospective study of thoracoscopic lobectomy, Maher et al found that the increased use of opioids within 6 hours after surgery was associated with a rise of recurrence rate in the patients with non-small cell lung cancer within 5 years after surgery. ${ }^{21}$ Hence, opioid requirement and administration dosage is directly related to unfavorable outcomes of lung cancer patients receiving perioperative analgesia. However, previous evidence for the use of opioids impact on the recurrence and metastasis of lung cancer cells are retrospective, thus, a large sample of prospective randomized controlled studies are needed to confirm that.

\section{Propofol and Etomidate}

Propofol and Etomidate play an important role in the sedation of patients undergoing cancer resection surgery or body check such as gastrointestinal endoscopy. There are discernible lung cancer inhibitory effects of Propofol
(2, 6-diisopropylphenol) in vitro and in vivo, and its mechanism of action remains comprehensive. Previous study found that Propofol suppressed viability, migration and invasion ability of human lung cancer A549 cells by targeting E-cadherin, N-cadherin, Vimentin and Snail expression. ${ }^{22}$ Another study pointed out that Propofol inhibits proliferation, migration and invasion ability of A549 cells by down-regulating microRNA (miR)-372, miR-1284 and miR-486, then inactivating Wnt/ $\beta$-catenin, mammalian target of rapamycin (mTOR) pathways and Propofol inhibits lung cancer A549 Cell Growth and Epithelial-Mesenchymal Transition Process, respectively. ${ }^{22-24}$ Moreover, another two studies demonstrated that Propofol suppressed lung cancer invasion via down-regulating aquaporin-3 (AQP-3), of the matrix metalloproteinase family such as matrix metalloproteinases (MMP-2, gelatinase A) and MMP-9 (gelatinase B), and attenuating the $\mathrm{p} 38$ mitogen-activated protein kinase (MAPK) signaling pathway. ${ }^{25,26}$ Additionally, Cui et al indicated that Propofol induced the expression of endoplasmic reticulum (ER) stress in lung cancer H460 cells and also significantly decreased the tumor volume and tumor weight of established xenografted human lung tumors. ${ }^{27}$ In clinical studies, Liu et al recruited 60 eligible candidates who underwent lung adenocarcinoma resection and were randomly divided into administering Propofol by target controlled infusion group, and administering Etomidate by continuous infusion to maintain the anesthetic state, respectively. ${ }^{28}$ Within the first $24 \mathrm{~h}$ after operation, CD4(+) T cells in the blood of the two groups of patients were significantly decreased, while CD8(+) T cells were increased compared with the preoperative state. The reduction level of CD4(+) T cells in Etomidate group were lower than that in the Propofol group. Thus, the immune function of adenocarcinoma patients in the Etomidate group was less affected than that in the Propofol group. ${ }^{28}$ However, there is no comparison of the long-term prognosis of patients using these two intravenous anesthetics. Further studies investigating the effects of Propofol on the recurrence and metastasis of lung cancer patients undergoing radical surgery are still needed.

\section{Lidocaine, Ropivacaine and Levobupivacaine}

Levobupivacaine induced epithelial-to-mesenchymal transition (EMT) in A549 lung cancer cells and caused the dissemination of lung cancer cells both in vitro and 
in vivo. Microarray, quantitative real-time polymerase chain reaction (qRT-PCR) and immunoblot analyses revealed that levobupivacaine upregulated the hypoxiainducible factor (HIF-2 $\alpha$ ) gene expression in cancer cells, which indicated that HIF- $2 \alpha$ signaling may contribute to levobupivacaine-mediated A549 cell dissemination. ${ }^{29}$ While in this study, there were no morphological changes observed in A549 cells after Lidocaine and Ropivacaine treatments. ${ }^{29}$ However, it was found by the in vitro studies of human lung adenocarcinoma cells that both Lidocaine and Ropivacaine, the amide local anesthetics, can reduce the migration of lung adenocarcinoma cells, but the ester local anesthetic, Chloroprocaine, does not have this function. The effects of Lidocaine and Ropivacaine are achieved by inhibiting important signaling pathways related to tumor growth and metastasis. These signaling pathways are characterized by the activation of Sre protein mediated by the tumor necrosis factor alpha (TNF- $\alpha$ ) and the phosphorylation of intercellular adhesion molecule 1 (ICAM-1). Piegeler et al further confirmed by in vitro studies that both Lidocaine and Ropivacaine could inhibit the growth and metastasis of human lung adenocarcinoma cells. ${ }^{30}$ Wang et al also found by in vitro studies of human non-small cell lung cancer that the mechanism of Lidocaine and Ropivacaine in inhibiting the growth and metastasis of lung cancer cells was mainly related to the apoptosis pathway and the MAPK pathway. ${ }^{31}$ These studies have explained to some extent the positive effect of general anesthesia combined with regional nerve block on the overall survival rate of lung cancer patients.

\section{Inhaled Anesthetics}

Inhaled anesthetics, such as Isoflurane and Sevoflurane, are inhaled via the lungs. These drugs subsequently pass through the alveolocapillary membrane and diffuse into the blood to induce anesthesia. In vitro experiments by Liang et al used Isoflurane or Sevoflurane separately in the anesthetic management of patients undergoing thoracoscopic radical resection of lung cancer. ${ }^{32}$ By detecting the number of glycoprotein II b/wa, CD62P and the platelet aggregation rate, it was found that the activation of platelets in patients who received Sevoflurane was lower than those who received Isoflurane. Researchers also found in lung adenocarcinoma cells cultured in vitro, that Sevoflurane could inhibit the growth of lung cancer cells by reducing the activity of platelets, while Isoflurane did not have such an effect. In vivo studies also found that the pretreatment of mouse lung cancer cells with Sevoflurane could inhibit metastasis, which might be related to the down-regulation of the expression of matrix metalloproteinases 2 and 9 that played a key role in tumor metastasis. There are many studies on the effects of inhaled anesthetics on the recurrence and metastasis of cancer cells, but there are few related studies about their effects on lung cancer cell recurrence and metastasis. ${ }^{33}$ More high-quality studies are required for confirmation.

\section{Non-Steroidal Anti-Inflammatory Drugs and Aspirin}

Non-steroidal anti-inflammatory drugs (NSAIDs) and aspirin are popular non-opioid analgesics for the treatment of pain after surgery. Previous study suggested that treatment with the NSAID nimesulide significantly increased the sensitivity of A549 cells to radiotherapy by inducing the cleavage of caspase-3, caspase-9, and poly (ADPribose) polymerase (PARP), activating caspase- 8 , and inducing cleavage of $\mathrm{Bid}^{34}$ In a retrospective cohort study of 347 patients with non-small cell lung cancer, the researchers found that postoperative NSAIDs and ibuprofen were associated with longer overall survival and tumor-free survival. ${ }^{35}$ Other studies also pointed out that perioperative inflammation was related to survival rate, and the application of ketorolac acid, diclofenac and Aspirin might increase survival rate. ${ }^{36}$ The dependent and independent mechanism of cyclooxygenase, a nonsteroidal anti-inflammatory drug, plays an important role in anti-tumorogenesis, but the exact mechanism is still a controversial issue. Further research should be made to explore the complex mechanisms.

\section{Intraoperative Blood Transfusion}

Intraoperative blood transfusion is also part of the anesthesiologist's work during lung cancer surgery. Cata et al pointed out in a retrospective study that the postoperative survival rate decreased in patients who underwent radical resection of stage I non-small cell lung cancer if they received allogeneic red blood cells intraoperatively or within 30 days postoperatively. ${ }^{37}$ In another retrospective study, it was found that although perioperative blood transfusion did not affect the survival rate of patients who underwent non-small cell lung cancer resection, blood transfusion was associated with a significant reduction in overall survival and tumor-free survival in a dosedependent manner. To avoid or minimize blood transfusion could improve the long-term survival rate of patients after 
lung cancer resection. ${ }^{38}$ Regarding the necessity for blood transfusion during radical resection of lung cancer, the anesthesiologist should make a comprehensive judgment based on the factors that affect the patient's oxygen supply and consumption such as the hemoglobin level, the cardiac function, and so on.

\section{Intraoperative Body Temperature}

Along with the development of the concept of enhanced recovery after surgery (ERAS), the management of body temperature during radical lung cancer surgery has drawn more and more attention from anesthesiologists. An in vitro study found that a low body temperature $\left(28^{\circ} \mathrm{C}\right)$ could activate human fat cells and promote the proliferation of human lung adenocarcinoma cells by increasing the levels of TNF-a secreted by fat cells or vascular endothelial cell growth factor (VECF). Another in vitro study pointed out that the three temperatures of $34{ }^{\circ} \mathrm{C}, 37{ }^{\circ} \mathrm{C}$, and $40{ }^{\circ} \mathrm{C}$ affected human lung adenocarcinoma cells in the way that mild hypothermia inhibited the proliferation of lung adenocarcinoma cells, while lung adenocarcinoma cells still exhibited a certain proliferation vitality at high temperatures within the fever range. ${ }^{39}$ The two in vitro studies mentioned above have reached the opposite conclusions of hypothermia on the proliferation of lung adenocarcinoma cells. The effect of intraoperative hypothermia on the recurrence and metastasis of lung cancer after surgery requires more evidence-based research.

\section{Operations Performed}

Recently, many large-scale single clinical observations have shown that Video-Assisted Thoracoscopic Surgery (VATS) lobectomy has an apparent advantage over the traditional thoracotomy in terms of postoperative pain relief, drainage time, and patients' postoperative comfort. $^{40-42}$ The National Comprehensive Cancer Network (NCCN) guideline (Version 8. 2017) has recommended VATS lobectomy as the preferred approach for early-stage NSCLC. However, there are still doubts about the use of VATS in locally advanced lung cancer. Multicenter researches are needed especially on the advantage of survival for patients underwent VATS lobectomy.

\section{Conclusion and Perspectives}

With the increasing incidence of lung cancer, there are more and more radical surgeries. Different management of anesthesia and narcotic substances may have different effects on the prognosis of patients undergoing radical surgeries for lung cancer (Figure 1). This paper reviewed the management of anesthesia in the recurrence and metastasis of lung cancer cells, including types of anesthesia, anesthetics, intraoperative

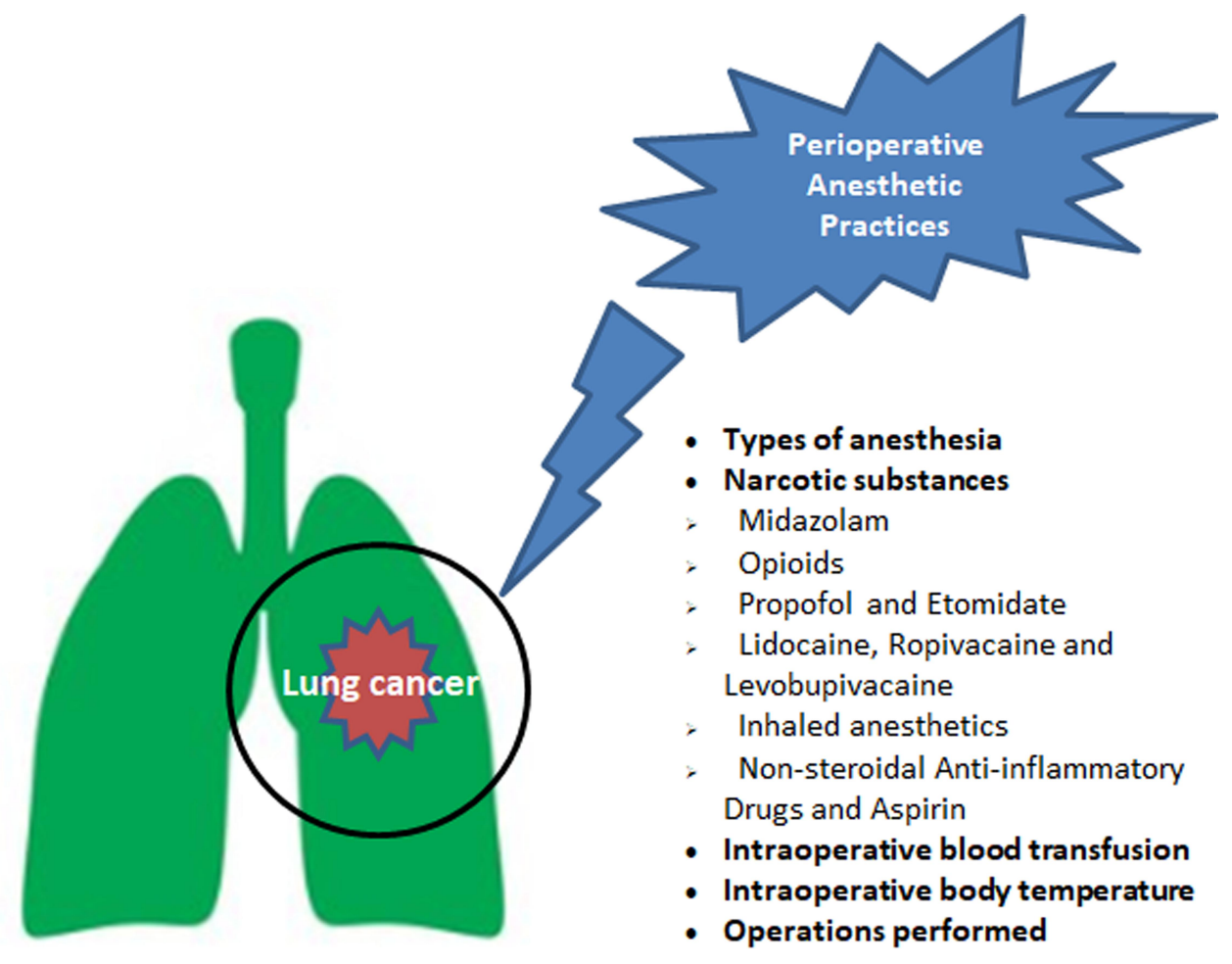

Figure I Association between perioperative anesthetic practices and lung cancer. 
blood transfusions and intraoperative body temperatures. Most studies involved were retrospective or in vitro, so they are of limited significance in guiding clinical anesthesia management. Besides, it is difficult to distinguish the contributions of specific drugs and the interactions between different techniques, because there is a variety of anesthetics and anesthetic techniques involved in anesthesia management. Therefore, randomized controlled prospective studies with a large clinical sample are needed to guide the anesthesia management in radical surgery for lung cancer. The mechanism of anesthesia management on the recurrence and metastasis of lung cancer should be further studied through basic experiments. Anesthesiologists should make great efforts to optimize anesthetic management, to both minimize the recurrence and metastasis of lung cancer in patients who undergo radical surgeries, and to improve their survival rate.

Generally speaking, perioperative anesthesia methods, analgesia techniques, anesthesia management and narcotic drug use may have a certain impact on the recurrence and migration of lung cancer. However, because the complexity of patients and the diversity of carcinogenic factors, it is impossible to separate the effects of anesthesia, surgery and other interference factors on lung cancer recurrence and metastasis. Therefore, based on current reports, there is no clear evidence that the change of single anesthesia technology can directly affect the long-term prognosis of patients. In addition, during the perioperative management, anesthesiologists should consider these above factors on patients with lung cancer and perform more studies to comprehensively evaluate the superposition effect of these factors in lung cancer.

\section{Abbreviations}

CNKI, China National Knowledge Infrastructure; TIVA, total intravenous anesthesia; NSCLC, non-small cell lung cancer; GPCRs, G protein-coupled receptors; 7TM, seven transmembrane domain; STAT3, signal transduction and activation of transcription 3; OGFR, opioid growth factor receptor; MMP, matrix metalloproteinases; EMT, epithelial-to-mesenchymal transition; HIF-2 $\alpha$, hypoxia-inducible factor; TNF- $\alpha$, tumor necrosis factor alpha; ICAM-1, intercellular adhesion molecule 1; MAPK, mitogen-activated protein kinase; PARP, poly ADP-ribose polymerase; ERAS, enhanced recovery after surgery.

\section{Funding}

This work was supported by the National Natural Science Foundation of China (No. 82003882), Natural Science
Foundation of Liaoning Province (No. 2019-ZD-0339), Science and Technology Projects of Shenyang (No. 19112-4-096), and Shenyang Young and Middle-aged Science and Technology Innovation Talents (No. RC200528).

\section{Disclosure}

The authors declare that the research was conducted in the absence of any commercial or financial relationships that could be construed as a potential conflict of interest.

\section{References}

1. Netto SM, Silva AC, Nunes RA, Gattass M. Voxel-based comparative analysis of lung lesions in CT for therapeutic purposes. Med Biol Eng Comput. 2017;55(2):295-314. doi:10.1007/s11517-016-1510-0

2. Xu Q, Shi NJ, Zhang H, Zhu YM. Effects of combined generalepidural anesthesia and total intravenous anesthesia on cellular immunity and prognosis in patients with non-small cell lung cancer: a comparative study. Mol Med Rep. 2017;16(4):4445-4454. doi:10.3892/mmr.2017.7144

3. Fu S, Qu PS, Cai SN. Effect of anesthetic methods on postoperative $\mathrm{CD} 3(+), \mathrm{CD} 4(+)$ and $\mathrm{CD} 4(+) \mathrm{CD} 25(+)$ in patients with lung cancer undergoing radical operation. Oncol Lett. 2018;16(5):6547-6551. doi:10.3892/ol.2018.9416

4. Cata JP, Bauer M, Sokari T, et al. Effects of surgery, general anesthesia, and perioperative epidural analgesia on the immune function of patients with non-small cell lung cancer. J Clin Anesth. 2013;25 (4):255-262. doi:10.1016/j.jclinane.2012.12.007

5. Lee EK, Ahn HJ, Zo JI, Kim K, Jung DM, Park JH. Paravertebral block does not reduce cancer recurrence, but is related to higher overall survival in lung cancer surgery: a retrospective cohort study. Anesth Analg. 2017;125(4):1322-1328. doi:10.1213/ANE.0000000000002342

6. Hollmann MW, Durieux ME, Fisher D. Local anesthetics and the inflammatory response: a new therapeutic indication? Anesthesiology. 2000;93:858-875. doi:10.1097/00000542-200009000-00038

7. Hollmann MW, Difazio CA, Durieux ME. Ca-signaling G-proteincoupled receptors: a new site of local anesthetic action? Reg Anesth Pain Med. 2001;26(6):565-571. doi:10.1053/rapm.2001.25923

8. Dorsam RT, Gutkind JS. G-protein-coupled receptors and cancer. Nat Rev Cancer. 2007;7(2):79-94. doi:10.1038/nrc2069

9. Venkatakrishnan AJ, Deupi X, Lebon G, Tate CG, Schertler GF, $\mathrm{Babu}$ MM. Molecular signatures of G-protein-coupled receptors. Nature. 2013;494(7436):185-194. doi:10.1038/nature11896

10. Horowitz M, Neeman E, Sharon E, Ben-Eliyahu S. Exploiting the critical perioperative period to improve long-term cancer outcomes. Nat Rev Clin Oncol. 2015;12(4):213-226. doi:10.1038/ nrclinonc.2014.224

11. Wigmore TJ, Mohammed K, Jhanji S. Long-term survival for patients undergoing volatile versus iv anesthesia for cancer surgery: a retrospective analysis. Anesthesiology. 2016;124(1):69-79. doi:10.1097/ALN.0000000000000936

12. Huang H, Benzonana LL, Zhao H, et al. Prostate cancer cell malignancy via modulation of HIF-1 $\alpha$ pathway with isoflurane and propofol alone and in combination. Br J Cancer. 2014;111(7):1338-1349. doi:10.1038/bjc.2014.426

13. Wang C, Datoo T, Zhao H, et al. Midazolam and dexmedetomidine affect neuroglioma and lung carcinoma cell biology in vitro and in vivo. Anesthesiology. 2018;129(5):1000-1014. doi:10.1097/ALN.00000000 00002401 
14. Jiao J, Wang Y, Sun X, Jiang X. Midazolam induces A549 cell apoptosis in vitro via the miR-520d-5p/STAT3 pathway. Int $J$ Clin Exp Pathol. 2018;11(3):1365-1373.

15. Jiao JH, Wang YH, Sun XF. Inhibitory effect and mechanism of midazolam on transplanted tumor of nude mice with human lung cancer A549 cells. J Clin Med Pract. 2020;24:21-25.

16. Schug SA, Chandrasena C. Pain management of the cancer patient. Expert Opin Pharmacother. 2015;16(1):5-15. doi:10.1517/ 14656566.2015.980723

17. Wang K, Zheng Y, Yang Y, et al. Nociceptin receptor is overexpressed in non-small cell lung cancer and predicts poor prognosis. Front Oncol. 2019;9:235. doi:10.3389/fonc.2019.00235

18. Singleton PA, Mirzapoiazova T, Hasina R, Salgia R, Moss J. Increased $\mu$-opioid receptor expression in metastatic lung cancer. $\mathrm{Br}$ J Anaesth. 2014;113(Suppl 1):i103-8. doi:10.1093/bja/aeu165

19. Kim JY, Ahn HJ, Kim JK, Kim J, Lee SH, Chae HB. Morphine suppresses lung cancer cell proliferation through the interaction with opioid growth factor receptor: an in vitro and human lung tissue study. Anesth Analg. 2016;123(6):1429-1436. doi:10.1213/ ANE.0000000000001293

20. Cata JP, Keerty V, Keerty D, et al. A retrospective analysis of the effect of intraoperative opioid dose on cancer recurrence after non-small cell lung cancer resection. Cancer Med. 2014;3 (4):900-908. doi:10.1002/cam4.236

21. Maher DP, Wong W, White PF, et al. Association of increased postoperative opioid administration with non-small-cell lung cancer recurrence: a retrospective analysis. Br J Anaesth. 2014;113(Suppl 1):i88-94. doi:10.1093/bja/aeu192

22. Liu WZ, Liu N. Propofol inhibits lung cancer A549 cell growth and epithelial-mesenchymal transition process by upregulation of MicroRNA-1284. Oncol Res. 2018;27(1):1-8. doi:10.3727/ 096504018X15172738893959

23. Sun H, Gao D. Propofol suppresses growth, migration and invasion of A549 cells by down-regulation of miR-372. BMC Cancer. 2018;18 (1):1252. doi:10.1186/s12885-018-5175-y

24. Yang N, Liang Y, Yang P, Yang T, Jiang L. Propofol inhibits lung cancer cell viability and induces cell apoptosis by upregulating microRNA-486 expression. Braz J Med Biol Res. 2017;50(1):e5794. doi:10.1590/1414-431x20165794

25. Wu KC, Yang ST, Hsia TC, et al. Suppression of cell invasion and migration by propofol are involved in down-regulating matrix metalloproteinase-2 and p38 MAPK signaling in A549 human lung adenocarcinoma epithelial cells. Anticancer Res. 2012;32(11):4833-4842.

26. Ye HJ, Bai JJ, Guo PP, Wang W, Lin CS. Propofol suppresses invasion of human lung cancer A549 cells by down-regulating aquaporin-3 and matrix metalloproteinase-9. Nan Fang $\mathrm{Yi} \mathrm{Ke} \mathrm{Da}$ Хие Хие Baо. 2016;36(9):1286-1290.

27. Cui WY, Liu Y, Zhu YQ, Song T, Wang QS. Propofol induces endoplasmic reticulum (ER) stress and apoptosis in lung cancer cell H460. Tumour Biol. 2014;35(6):5213-5217. doi:10.1007/s13277014-1677-7

28. Liu J, Dong W, Wang T, et al. Effects of etomidate and propofol on immune function in patients with lung adenocarcinoma. Am J Transl Res. 2016;8(12):5748-5755.

29. Chan SM, Lin BF, Wong CS, Chuang WT, Chou YT, Wu ZF. Levobuipivacaine-induced dissemination of A549 lung cancer cells. Sci Rep. 2017;7(1):8646. doi:10.1038/s41598-017-08885-z

30. Piegeler T, Schläpfer M, Dull RO, et al. Clinically relevant concentrations of lidocaine and ropivacaine inhibit TNF $\alpha$-induced invasion of lung adenocarcinoma cells in vitro by blocking the activation of Akt and focal adhesion kinase. Br J Anaesth. 2015;115(5):784-791. doi:10.1093/bja/aev341
31. Wang HW, Wang LY, Jiang L, Tian SM, Zhong TD, Fang XM. Amide-linked local anesthetics induce apoptosis in human non-small cell lung cancer. J Thorac Dis. 2016;8(10):2748-2757. doi:10.21037/jtd.2016.09.66

32. Liang $\mathrm{H}$, Yang $\mathrm{CX}$, Zhang $\mathrm{B}$, Zhao ZL, Zhong JY, Wen XJ. Sevoflurane attenuates platelets activation of patients undergoing lung cancer surgery and suppresses platelets-induced invasion of lung cancer cells. J Clin Anesth. 2016;35:304-312. doi:10.1016/j. jclinane.2016.08.008

33. Benzonana LL, Perry NJ, Watts HR, et al. Isoflurane, a commonly used volatile anesthetic, enhances renal cancer growth and malignant potential via the hypoxia-inducible factor cellular signaling pathway in vitro. Anesthesiology. 2013;119(3):593-605. doi:10.1097/ ALN.0b013e31829e47fd

34. Kim BM, Won J, Maeng KA, Han YS, Yun YS, Hong SH. Nimesulide, a selective COX-2 inhibitor, acts synergistically with ionizing radiation against A549 human lung cancer cells through the activation of caspase-8 and caspase-3. Int J Oncol. 2009;34 (5):1467-1473.

35. Jiang W, Wang L, Zhang $J$, et al. Effects of postoperative non-steroidal anti-inflammatory drugs on long-term survival and recurrence of patients with non-small cell lung cancer. Medicine (Baltimore). 2018;97(39):e12442. doi:10.1097/ MD.0000000000012442

36. Izquierdo E, Fábregas N, Valero R, Salvador L, Soley R, Nalda MA. Postoperative analgesia in herniated disk surgery. Comparative study of diclofenac, lysine acetylsalicylate, and ketorolac. Rev Esp Anestesiol Reanim. 1995;42(8):316-319.

37. Cata JP, Gutierrez C, Mehran RJ, et al. Preoperative anemia, blood transfusion, and neutrophil-to-lymphocyte ratio in patients with stage i non-small cell lung cancer. Cancer Cell Microenviron. 2016;3(1): e1116. doi: $10.14800 / \mathrm{ccm} .1116$

38. Latif MJ, Tan KS, Molena D, et al. Perioperative blood transfusion has a dose-dependent relationship with disease recurrence and survival in patients with non-small cell lung cancer. $J$ Thorac Cardiovasc Surg. 2019;157(6):2469-77.e10. doi:10.1016/j.jtcvs.2018.12.109

39. Kalamida D, Karagounis IV, Mitrakas A, Kalamida S, Giatromanolaki A, Koukourakis MI. Fever-range hyperthermia vs. hypothermia effect on cancer cell viability, proliferation and HSP90 expression. PLoS One. 2015;10(1):e0116021. doi:10.1371/journal. pone. 0116021

40. Onaitis MW, Petersen RP, Balderson SS, Toloza E, Burfeind WR, Harpole DH Jr. Thoracoscopic lobectomy is a safe and versatile procedure: experience with 500 consecutive patients. Ann Surg. $2006 ; 244(3): 420-425$. doi:10.1097/01.sla.0000234892.7905 6.63

41. Swanson SJ, Herndon JE, D’Amico TA, Demmy TL. Video-assisted thoracic surgery lobectomy: report of CALGB 39802-a prospective, multi-institution feasibility study. $J$ Clin Oncol. 2007;25 (31):4993-4997. doi:10.1200/JCO.2007.12.6649

42. Whitson BA, Andrade RS, Boettcher A, et al. Video-assisted thoracoscopic surgery is more favorable than thoracotomy for resection of clinical stage I non-small cell lung cancer. Ann Thorac Surg. 2007;83(6):1965-1970. doi:10.1016/j.athoracsur. 2007.01.049 


\section{Publish your work in this journal}

Drug Design, Development and Therapy is an international, peerreviewed open-access journal that spans the spectrum of drug design and development through to clinical applications. Clinical outcomes, patient safety, and programs for the development and effective, safe, and sustained use of medicines are a feature of the journal, which has also been accepted for indexing on PubMed Central. The manuscript management system is completely online and includes a very quick and fair peer-review system, which is all easy to use. Visit http://www. dovepress.com/testimonials.php to read real quotes from published authors. 